Network Working Group

Request for Comments: 1523

Category: Informational
N. Borenstein

Bellcore

September 1993

The text/enriched MIME Content-type

Status of this Memo

This memo provides information for the Internet community. It does not specify an Internet standard. Distribution of this memo is unlimited.

Abstract

MIME [RFC-1341, RFC-1521] defines a format and general framework for the representation of a wide variety of data types in Internet mail. This document defines one particular type of MIME data, the text/enriched type, a refinement of the "text/richtext" type defined in RFC 1341. The text/enriched MIME type is intended to facilitate the wider interoperation of simple enriched text across a wide variety of hardware and software platforms.

The Text/enriched MIME type

In order to promote the wider interoperability of simple formatted text, this document defines an extremely simple subtype of the MIME content-type "text", the "text/enriched" subtype. This subtype was designed to meet the following criteria:

1. The syntax must be extremely simple to parse, so that even teletype-oriented mail systems can easily strip away the formatting information and leave only the readable text.

2. The syntax must be extensible to allow for new formatting commands that are deemed essential for some application.

3. If the character set in use is ASCII or an 8- bit ASCII superset, then the raw form of the data must be readable enough to be largely unobjectionable in the event that it is displayed on the screen of the user of a non-MIME-conformant mail reader.

4. The capabilities must be extremely limited, to ensure that it can represent no more than is likely to be representable by the user's primary word processor. While this limits what can be sent, it increases the likelihood that what is sent can be properly displayed. 
This document defines a new MIME content-type, "text/enriched". The content-type line for this type may have one optional parameter, the "charset" parameter, with the same values permitted for the "text/plain" MIME content-type.

The syntax of "text/enriched" is very simple. It represents text in a single character set -- US-ASCII by default, although a different character set can be specified by the use of the "charset" parameter. (The semantics of text/enriched in non-ASCII character sets are discussed later in this document.) All characters represent themselves, with the exception of the "<" character (ASCII 60), which is used to mark the beginning of a formatting command. Formatting instructions consist of formatting commands surrounded by angle brackets ("<>", ASCII 60 and 62). Each formatting command may be no more than 60 characters in length, all in US-ASCII, restricted to the alphanumeric and hyphen ("-") characters. Formatting commands may be preceded by a solidus ("/", ASCII 47), making them negations, and such negations must always exist to balance the initial opening commands. Thus, if the formatting command "<bold>" appears at some point, there must later be a "</bold $>$ " to balance it. (NOTE: The 60 character limit on formatting commands does NOT include the "<", ">", or "/" characters that might be attached to such commands.)

Formatting commands are always case-insensitive. That is, "bold" and "BoLd" are equivalent in effect, if not in good taste.

Beyond tokens delimited by "<" and ">", there are two other special processing rules. First, a literal less-than sign ("<") can be represented by a sequence of two such characters, $"<<"$. Second, line breaks (CRLF pairs in standard network representation) are handled specially. In particular, isolated CRLF pairs are translated into a single SPACE character. Sequences of N consecutive CRLF pairs, however, are translated into N-1 actual line breaks. This permits long lines of data to be represented in a natural- looking manner despite the frequency of line-wrapping in Internet mailers. When preparing the data for mail transport, isolated line breaks should be inserted wherever necessary to keep each line shorter than 80 characters. When preparing such data for presentation to the user, isolated line breaks should be replaced by a single SPACE character, and $\mathrm{N}$ consecutive CRLF pairs should be presented to the user as $\mathrm{N}-1$ line breaks. 
Thus text/enriched data that looks like this:

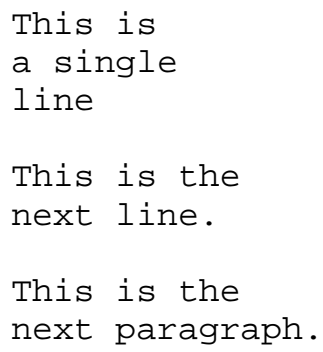


Smaller -- causes the affected text to be in a smaller font. It is recommended that the font size be changed by two points, but other amounts may be more appropriate in some environments. Nested smaller commands produce eversmaller fonts, to the limits of the implementation's capacity to reasonably display them, after which further smaller commands have no incremental effect.

Bigger -- causes the affected text to be in a bigger font. It is recommended that the font size be changed by two points, but other amounts may be more appropriate in some environments. Nested bigger commands produce ever-bigger fonts, to the limits of the implementation's capacity to reasonably display them, after which further bigger commands have no incremental effect.

Underline -- causes the affected text to be underlined. Nested underline commands have the same effect as a single underline command.

While the "bigger" and "smaller" operators are effectively inverses, it is not recommended, for example, that "<smaller>" be used to end the effect of "<bigger>". This is properly done with "</bigger>".

Justification Commands

Initially, text/enriched text is intended to be displayed fullyjustified with appropriate fill, kerning, and letter-tracking as suits the capabilities of the receiving user agent software. Actual line width is left to the discretion of the receiver, which is expected to fold lines intelligently (preferring soft line breaks) to the best of its ability.

The following commands alter that state. Each of these commands force a line break before and after the formatting command if there is not otherwise a line break. For example, if one of these commands occurs anywhere other than the beginning of a line of text as presented, a new line is begun.

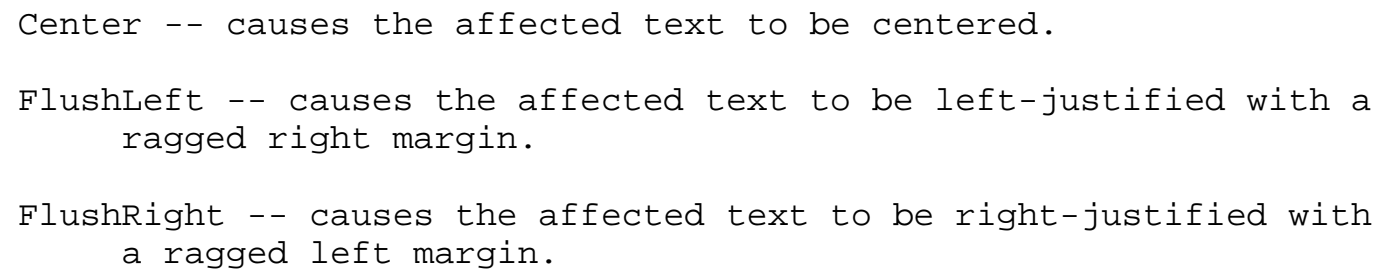


The center, flushleft, and flushright commands are mutually exclusive, and, when nested, the inner command takes precedence.

Note that for some non-ASCII character sets, full justification may be inappropriate. In these cases, a user agent may choose not to justify such data.

\section{Indentation Commands}

Initially, text/enriched text is displayed using the maximum available margins. Two formatting commands may be used to affect the margins.

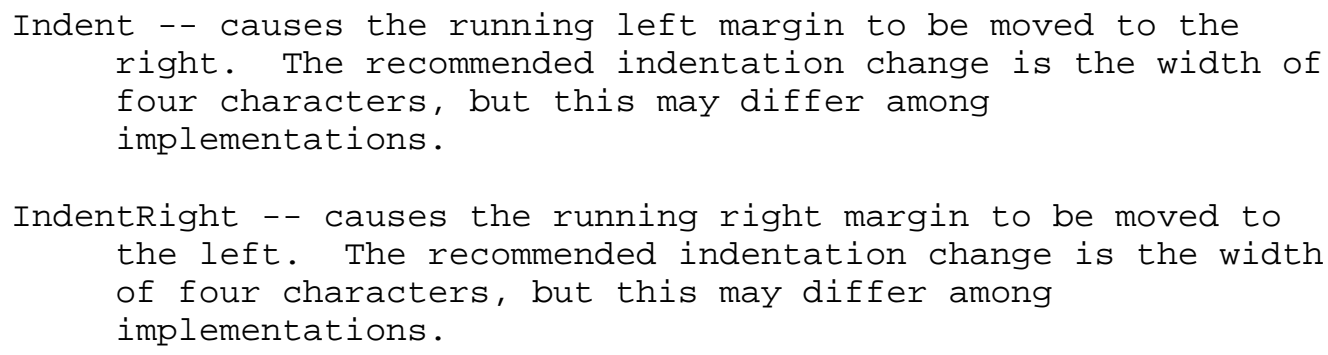

A line break is NOT forced by a change of the margin, to permit the description of "hanging" text. Thus for example the following text:

Now <indent> is the time for all good horses to come to the aid of their stable, assuming that </indent> any stable is really stable.

would be displayed in a 40-character-wide window as follows:

Now is the time for all good horses to come to the aid of their stable, assuming that any stable is really stable.

Miscellaneous Commands

Excerpt -- causes the affected text to be interpreted as a textual excerpt from another source, probably a message being responded to. Typically this will be displayed using indentation and an alternate font, or by indenting lines and preceding them with "> ", but such decisions are up to the implementation. (Note that this is the only truly declarative markup construct in text/enriched, and as such doesn't fit very well with the other facilities, but it describes a type of markup that is very commonly used in email and has no procedural analogue.) Note that 
as with the justification commands, the excerpt command implicitly begins and ends with a line break if one is not already there.

Verbatim -- causes the affected text to be displayed without filling, justification, any interpretation of embedded formatting commands, or the usual special rules for CRLF handling. Note, however, that the end token </verbatim> must still be recognized.

Nofill -- causes the affected text to be displayed without filling or justification, and hence without any special handling of CRLFs, but with all remaining text/enriched features continuing to apply.

Param -- Marks the affected text as command parameters, to be interpreted or ignored by the text/enriched interpreter, but NOT to be shown to the reader.

Note that while the absence of a quoting mechanism makes it slightly challenging to include the literal string "<verbatim>" inside of a verbatim environment, it can be done by breaking up the verbatim segment into two verbatim segments as follows:

<verbatim>

...slightly challenging to include the literal string

$"</</$ verbatim><verbatim>verbatim>" inside of a verbatim

environment...

$</$ verbatim $>$

Note that the above example demonstrates that it is not desirable for an implementation to break lines between tokens. In particular, there should not be a line break inserted between the "</verbatim>" and the "<verbatim>" that follows it.

Balancing and Nesting of Formatting Commands

Pairs of formatting commands must be properly balanced and nested. Thus, a proper way to describe text in bold italics is:

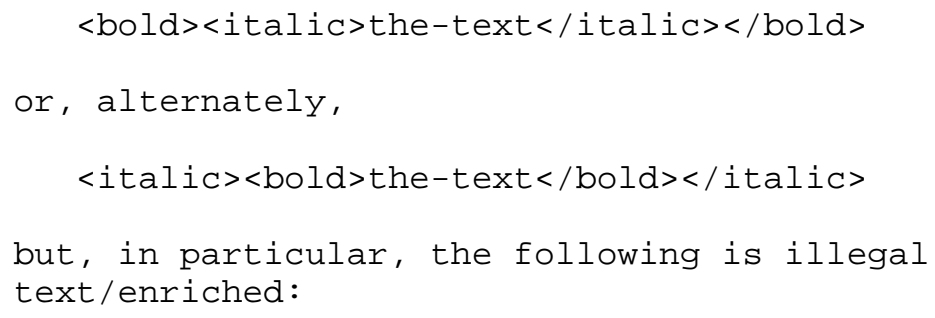


$<$ bold $><$ italic $>$ the-text $</$ bold $></$ italic $>$

The nesting requirement for formatting commands imposes a slightly higher burden upon the composers of text/enriched bodies, but potentially simplifies text/enriched displayers by allowing them to be stack-based. The main goal of text/enriched is to be simple enough to make multifont, formatted email widely readable, so that those with the capability of sending it will be able to do so with confidence. Thus slightly increased complexity in the composing software was deemed a reasonable tradeoff for simplified reading software. Nonetheless, implementors of text/enriched readers are encouraged to follow the general Internet guidelines of being conservative in what you send and liberal in what you accept. Those implementations that can do so are encouraged to deal reasonably with improperly nested text/enriched data.

Unrecognized formatting commands

Implementations must regard any unrecognized formatting command as "no-op" commands, that is, as commands having no effect, thus facilitating future extensions to "text/enriched". Private extensions may be defined using formatting commands that begin with "X-", by analogy to Internet mail header field names.

In order to formally define extended commands, a new Internet document should be published.

"White space" in text/enriched Data

No special behavior is required for the SPACE or TAB (HT) character. It is recommended, however, that, at least when fixed-width fonts are in use, the common semantics of the TAB (HT) character should be observed, namely that it moves to the next column position that is a multiple of 8. (In other words, if a TAB (HT) occurs in column $n$, where the leftmost column is column 0 , then that TAB (HT) should be replaced by 8-( $\mathrm{n}$ mod 8) SPACE characters.) It should also be noted that some mail gateways are notorious for losing (or, less commonly, adding) white space at the end of lines, so reliance on SPACE or TAB characters at the end of a line is not recommended.

Initial state of a text/enriched interpreter

Text/enriched is assumed to begin with filled, fully justified text in a variable-width font in a normal typeface and a size that is average for the current display and user. The left and right margins are assumed to be maximal, that is, at the leftmost and rightmost acceptable positions. 
Non-ASCII character sets

If the character set specified by the charset parameter on the Content-type line is anything other than "US-ASCII", this means that the text being described by text/enriched formatting commands is in a non-ASCII character set. However, the commands themselves are still the same ASCII commands that are defined in this document. This creates an ambiguity only with reference to the "<" character, the octet with numeric value 60. In single byte character sets, such as the ISO-8859 family, this is not a problem; the octet 60 can be quoted by including it twice, just as for ASCII. The problem is more complicated, however, in the case of multi-byte character sets, where the octet 60 might appear at any point in the byte sequence for any of several characters.

In practice, however, most multibyte character sets address this problem internally. For example, the ISO-2022 family of character sets can switch back into ASCII at any moment. Therefore it is specified that, before text/enriched formatting commands, the prevailing character set should be "switched back" into ASCII, and that only those characters which would be interpreted as "<" in plain text should be interpreted as token delimiters in text/enriched.

The question of what to do for hypothetical future character sets that do NOT subsume ASCII is not addressed in this memo.

Minimal text/enriched conformance

A minimal text/enriched implementation is one that simply recognizes the beginning and ending of "verbatim" environments and, outside of them, converts "<<" to "<", removes everything between a <param> command and the next balancing </param> command, removes all other formatting commands (all text enclosed in angle brackets), converts any series of $n$ CRLFs to $n-1$ CRLFs, and converts any lone CRLF pairs to SPACE.

Notes for Implementors

It is recognized that implementors of future mail systems will want rich text functionality far beyond that currently defined for text/enriched. The intent of text/enriched is to provide a common format for expressing that functionality in a form in which much of it, at least, will be understood by interoperating software. Thus, in particular, software with a richer notion of formatted text than text/enriched can still use text/enriched as its basic representation, but can extend it with new formatting commands and by hiding information specific to that software system in text/enriched <param> constructs. As such systems evolve, it is expected that the 
definition of text/enriched will be further refined by future published specifications, but text/enriched as defined here provides a platform on which evolutionary refinements can be based.

An expected common way that sophisticated mail programs will generate text/enriched data is as part of a multipart/alternative construct. For example, a mail agent that can generate enriched mail in ODA format can generate that mail in a more widely interoperable form by generating both text/enriched and ODA versions of the same data, e.g.:

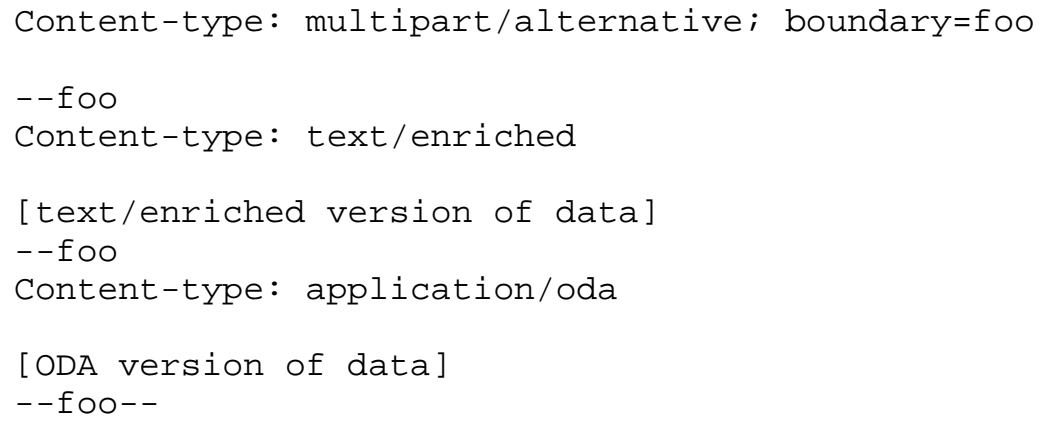

If such a message is read using a MIME-conformant mail reader that understands ODA, the ODA version will be displayed; otherwise, the text/enriched version will be shown.

In some environments, it might be impossible to combine certain text/enriched formatting commands, whereas in others they might be combined easily. For example, the combination of <bold> and <italic> might produce bold italics on systems that support such fonts, but there exist systems that can make text bold or italicized, but not both. In such cases, the most recently issued (innermost) recognized formatting command should be preferred.

One of the major goals in the design of text/enriched was to make it so simple that even text-only mailers will implement enriched-toplain-text translators, thus increasing the likelihood that enriched text will become "safe" to use very widely. To demonstrate this simplicity, an extremely simple C program that converts text/enriched input into plain text output is included in Appendix A.

Extensions to text/enriched

It is expected that various mail system authors will desire extensions to text/enriched. The simple syntax of text/enriched, and the specification that unrecognized formatting commands should simply be ignored, are intend to promote such extensions. 


\begin{abstract}
Beyond simply defining new formatting commands, however, it may sometimes be necessary to define formatting commands that can take arguments. This is the intended use of the <param> construct. In particular, software that wished to extend text/enriched to include colored text might define an "x-color" environment which always began with a color name parameter, to indicate the desired color for the affected text.
\end{abstract}

An Example

Putting all this together, the following "text/enriched" body fragment:

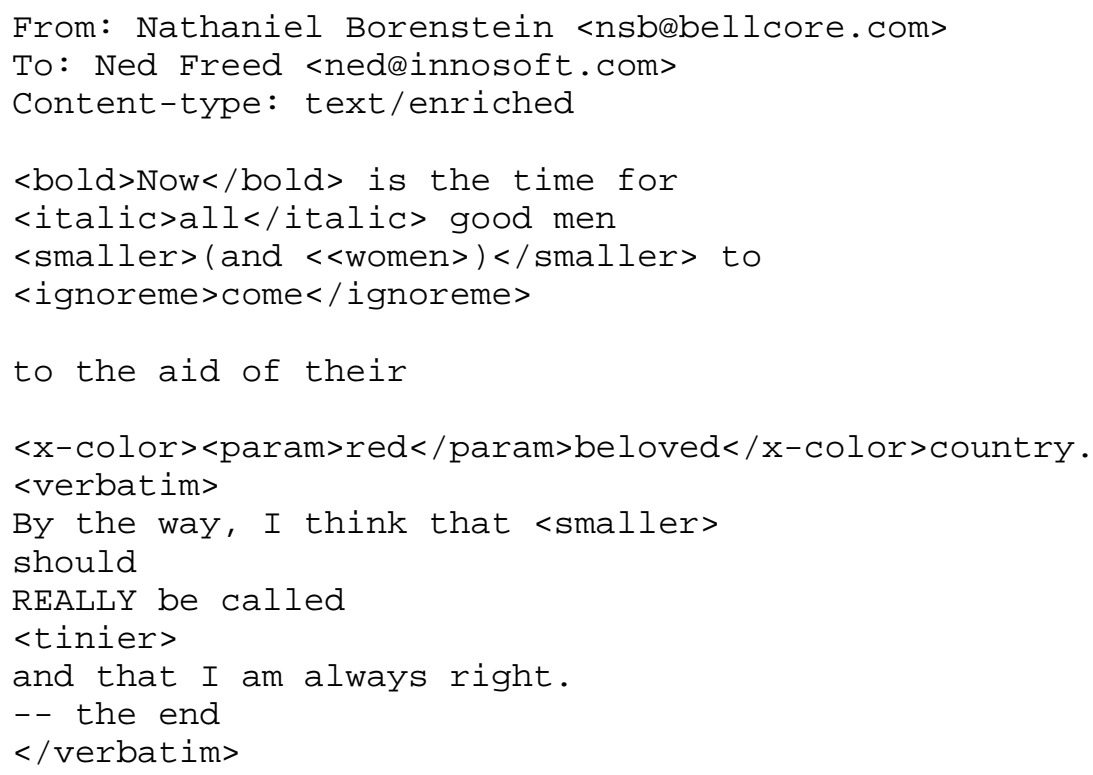


where the word "beloved" would be in red on a color display if the receiving software implemented the "x-color" extension.

Security Considerations

Security issues are not discussed in this memo, as the mechanism raises no security issues.

Author's Address

For more information, the author of this document may be contacted via Internet mail:

Nathaniel S. Borenstein

MRE 2D-296, Bellcore

445 South st.

Morristown, NJ 07962-1910

Phone: +12018294270

Fax: +1 2018295963

EMail: nsbabellcore.com

Acknowledgements

This document reflects the input of many contributors, readers, and implementors of the original MIME specification, RFC 1341. This memo also reflects particular contributions and comments from Terry Crowley and Rhys Weatherley. 
Appendix A -- A Simple enriched-to-plain Translator in C

One of the major goals in the design of the text/enriched subtype of the text Content-Type is to make formatted text so simple that even text-only mailers will implement enriched-to-plain-text translators, thus increasing the likelihood that multifont text will become "safe" to use very widely. To demonstrate this simplicity, what follows is a simple C program that converts text/enriched input into plain text output. Note that the local newline convention (the single character represented by " $\backslash \mathrm{n} "$ ) is assumed by this program, but that special CRLF handling might be necessary on some systems.

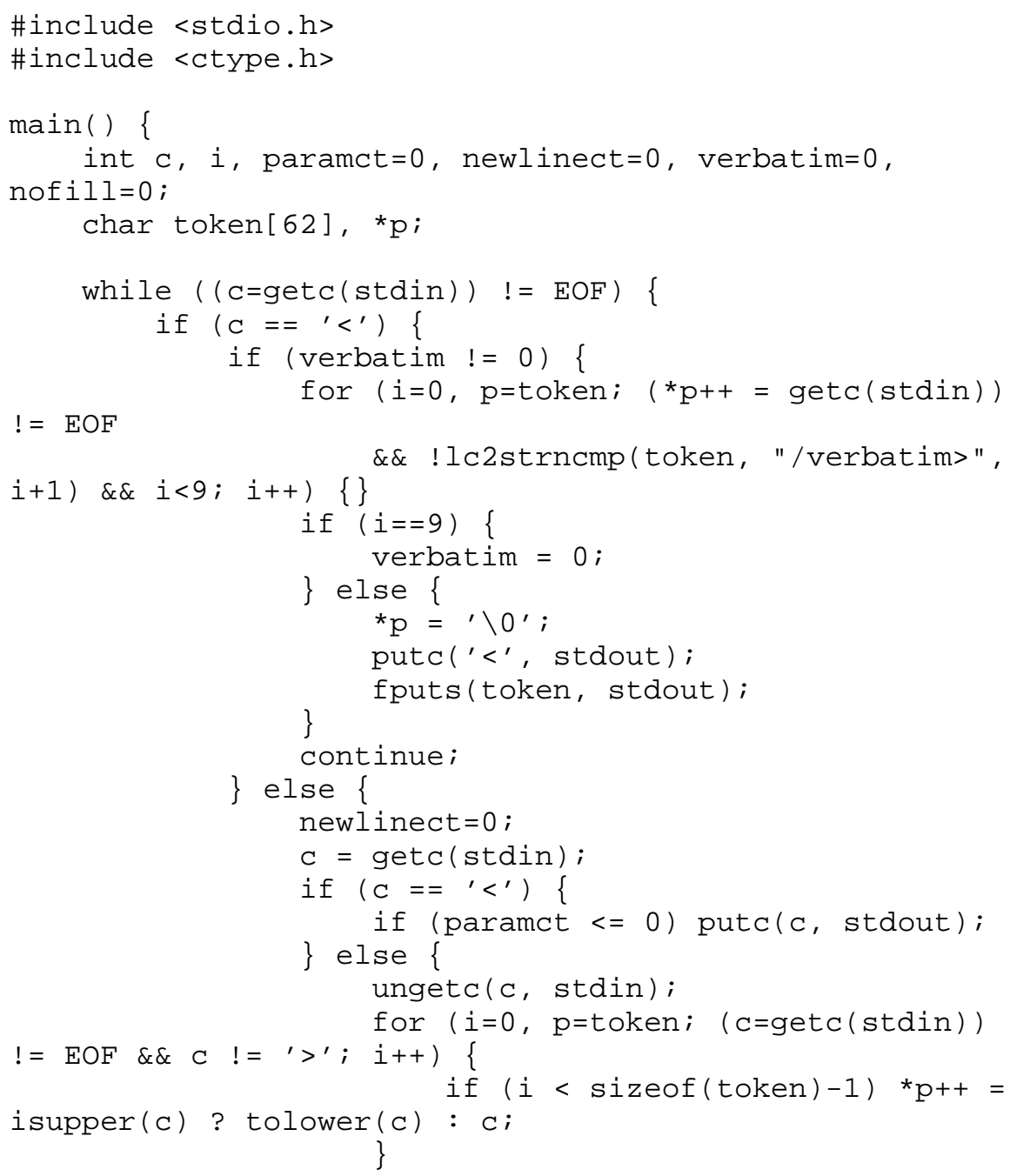




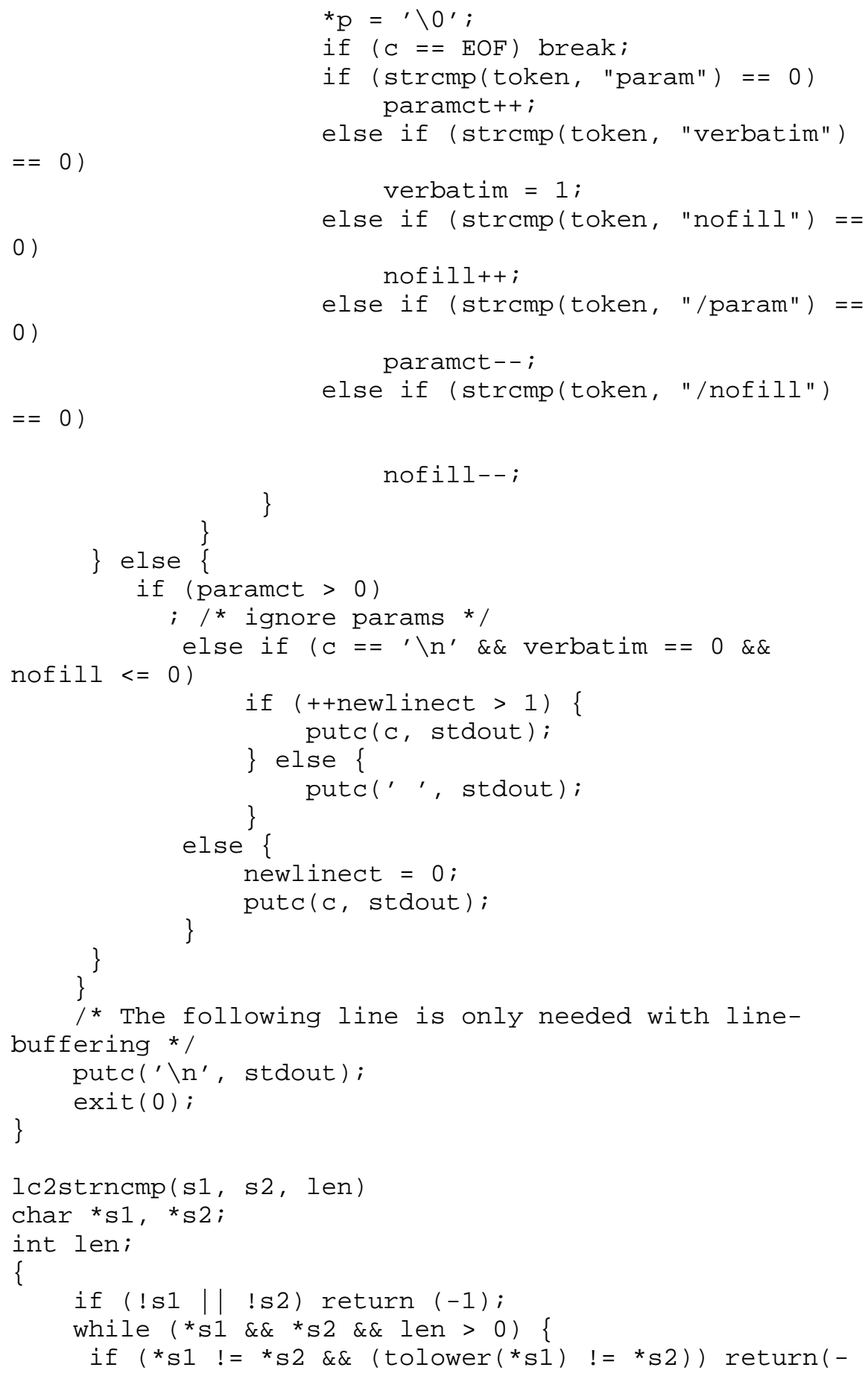


1) ;

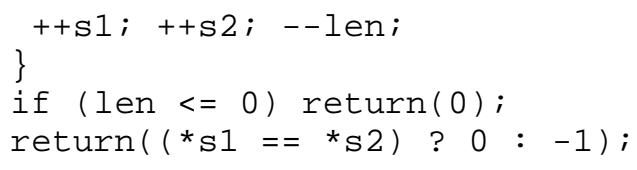

It should be noted that one can do considerably better than this in displaying text/enriched data on a dumb terminal. In particular, one can replace font information such as "bold" with textual emphasis (like *this* or _T_H_I_S_). One can also properly handle the text/enriched formatting commands regarding indentation, justification, and others. However, the above program is all that is necessary in order to present text/enriched on a dumb terminal without showing the user any formatting artifacts.

Appendix B -- Differences from RFC 1341 text/richtext

Text/enriched is a clarification, simplification, and refinement of the type defined as text/richtext in RFC 1341. For the benefit of those who are already familiar with text/richtext, or for those who want to exploit the similarities to be able to display text/richtext data with their text/enriched software, the differences between the two are summarized here. Note, however, that text/enriched is intended to make text/richtext obsolete, so it is not recommended that new software generate text/richtext.

0 . The name "richtext" was changed to "enriched", both to differentiate the two versions and because "richtext" created widespread confusion with Microsoft's Rich Text Format (RTF).

1. Clarifications. Many things were ambiguous or unspecified in the text/richtext definition, particularly the initial state and the semantics of richtext with multibyte character sets. However, such differences are OPERATIONALLY irrelevant, since the clarifications offered in this document are at least reasonable interpretations of the text/richtext specification.

2. Newline semantics have changed. In text/richtext, all CRLFs were mapped to spaces, and line breaks were indicated by "<nl>". This has been replaced by the "n-1" rule for CRLFs.

3. The representation of a literal "<" character was "<lt>" in text/richtext, but is "<<" in text/enriched.

4. The "verbatim" and "nofill" commands did not exist in text/richtext. 
5. The "param" command did not exist in text/richtext.

6. The following commands from text/richtext have been REMOVED from text/enriched: <COMMENT>, <OUTDENT>, <OUTDENTRIGHT>, <SAMEPAGE>, $<$ SUBSCRIPT>, <SUPERSCRIPT>, <HEADING>, <FOOTING>, <ISO-8859-[1-9]>,

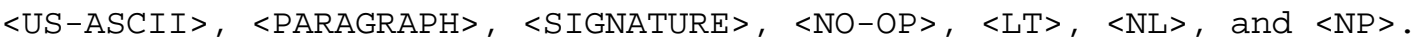

7. All claims of SGML compatibility have been dropped. However, with the possible exceptions of the new semantics for CRLF and " $<<"$ can be implemented, text/enriched should be no less SGML-friendly than text/richtext was.

8. In text/richtext, there were three commands $(\langle\mathrm{NL}\rangle,\langle\mathrm{NP}\rangle$, and $\langle\mathrm{LT}>$ ) that did not use balanced closing delimiters. Since all of these have been eliminated, there are No exceptions to the nesting/balancing rules in text/enriched.

9. The limit on the size of formatting tokens has been increased from 40 to 60 characters.

References

[RFC-1341] Borenstein, N., and N. Freed, "MIME (Multipurpose Internet Mail Extensions): Mechanisms for Specifying and Describing the Format of Internet Message Bodies", RFC 1341, Bellcore, Innosoft, June 1992.

[RFC-1521] Borenstein, N., and N. Freed, "MIME (Multipurpose Internet Mail Extensions) Part One: Mechanisms for Specifying and Describing the Format of Internet Message Bodies", RFC 1521, September 1993. 